\title{
Adaptive local binary pattern with oriented standard deviation (ALBPS) for texture classification
}

\author{
Oscar García-Olalla*, Enrique Alegre, Laura Fernández-Robles, María Teresa García-Ordás \\ and Diego García-Ordás
}

\begin{abstract}
A new method to describe texture images using a hybrid combination of local and global texture descriptors is proposed in this paper. In this regard, a new adaptive local binary pattern (ALBP) descriptor is presented in order to carry out the local description. It is built by adding oriented standard deviation information to an ALBP descriptor in order to achieve a more complete representation of the images, and hence, it has been called adaptive local binary pattern with oriented standard deviation (ALBPS). Regarding semen vitality assessment, ALBPS outperformed previous literature works with an $81.88 \%$ accuracy and also yielded higher hit rates than the LBP and ALBP baseline methods. Concerning the global description of the images, several classical texture algorithms were tested and a descriptor based on wavelet transform and Haralick feature extraction (wavelet concurrent feature 13 (WCF13)) obtained the best results. Both local and global descriptors were combined, and the classification was carried out with a support vector machine. Two data sets have been evaluated: textures under varying illumination, pose and scale (KTH-TIPS) 2a data set and a second spermatozoa boar data set used to distinguish between dead or alive sperm heads. Therefore, our proposal is novel in three ways. First, a new local feature extraction method ALBPS is introduced. Second, a hybrid method combining the proposed local ALBPS and a global descriptor is presented, outperforming our first approach and all other methods evaluated for this problem. Third, texture classification accuracy is greatly improved with the two former texture descriptors presented. F score and accuracy values were computed in order to measure the performance. The best overall result was yielded by combining ALBPS with WCF13, reaching an F score $=0.886$ and an accuracy of $85.63 \%$ in the spermatozoa data set and an $84.45 \%$ of hit rate in the KTH-TIPS 2 a.
\end{abstract}

Keywords: Texture analysis, Image processing, LBP, Adaptivity

\section{Introduction}

Texture analysis is a basic vision problem which has been thoroughly studied in the last decades [1]. Its numerous applications vary from object recognition to content-based image retrieval. The first works in texture classification focus on the statistical analysis of images. Some representative methods [2-4] rely on texture features determined from the grey level co-occurrence matrix (GLCM) by Haralick [5], while others are based on Gabor filter bank responses, one of the most prevailing filters [6].

\footnotetext{
*Correspondence: ogaro@unileon.es
} University of Leon, Leon 24071, Spain
Local binary patterns (LBP), proposed by Ojala et al. [7], are a recent method commonly used to describe texture. We have chosen to use this approach as a baseline in order to classify texture because it is a very descriptive and efficient texture operator while really simple. LBP just labels the pixels of an image by thresholding the neighbourhood of each pixel and considers the result as a binary number. Due to its computational simplicity and discriminative power, LBP has become a popular approach in several applications such as texture classification and segmentation, face recognition or visual inspection, specially in challenging real-time settings. Moreover, many variants have been proposed in order to improve its performance in terms of, for example, rotation invariant [8], robustness on flat images [9] or color invariance [10].

\section{黑 Springer}

C 2013 García-Olalla et al.; licensee Springer. This is an Open Access article distributed under the terms of the Creative Commons Attribution License (http://creativecommons.org/licenses/by/2.0), which permits unrestricted use, distribution, and reproduction in any medium, provided the original work is properly cited. 
In many practical applications, textures are captured in arbitrary orientations. Lately, Guo and his colleagues have developed several methods which modify an LBP operator in order to improve its performance and robustness to rotation. In their study [11], Guo et al. used the oriented standard deviation information of the neighbourhood in the matching step to obtain rotation invariance. They called it adaptive LBP (ALBP) approach. Recently, a new method which considers different sizes of the neighbourhood in order to create a unique feature vector for rotation invariance has been presented in one study of Guo et al. [12], outperforming their other study [11]. Besides, Guo et al. [13] introduced a novel approach to improve features extraction in images by varying the uniformity criterion of the patterns of the image through LBP.

Following the idea of Guo et al., which is to achieve rotation invariance [11], García-Olalla et al., [14] presented an approach called adaptive local binary pattern with oriented standard deviation (ALBPS), which adds an oriented standard deviation term to the LBP operator instead of using this information in the matching. ALBPS proved to obtain better performance than ALBP when assessing boar sperm vitality, plus it allowed to use a support vector machine (SVM) classifier, improving computational time with respect to the $1 \times 1$ matching algorithm proposed in ALBP. Furthermore, results when using a combination of ALBPS with global texture descriptors outperformed the methods individually. In this paper, we ascertain that ALBPS achieves good results in a more general context apart from vitality assessment.

In the context of texture categorization, several data sets have been proposed to evaluate the descriptors. In their study [15], Caputo et al. proposed a challenging data set for material recognition called textures under varying illumination, pose and scale (KTH-TIPS) $2 \mathrm{a}$ and a methodology to evaluate it. A lot of researchers have tested their work using this data set. One example is the work developed by Chen et al. [16] proposing a robust local image descriptor called WLD. KTH-TIPS 2a data set is used in our work to test ALBPS, broadening its applications for generic texture classification tasks.

In regard to vitality assessment, few works deal with evaluating the vitality of a sample in order to classify the spermatozoa heads as dead or alive. Alegre et al. $[17,18]$ proposed a method using texture descriptors that obtained a $76.80 \%$ hit rate when testing images captured at $\times 100$. Sperm assessment is used in the porcine industry for artificial insemination, so the quality of the semen must be higher as possible. A vision approach allows to assess the semen vitality without fluorescent stains which implies a great decrease in time and money.
The outline of the paper is as follows: descriptors, patterns and the proposed method are described in the "Methodology" section. The two data sets, experiment set-up and achieved results with the proposed descriptors are presented in the "Experiments" section, and finally, there is the "Conclusions" section.

\section{Methodology}

\section{Global texture descriptors}

In our first approach, the first data set, composed of images showing boar spermatozoa heads, has been characterised by several global texture descriptors. Seven classical texture descriptors have been computed for each image. The first one was a feature vector made up of four statistical measures of texture taken from the greyscale original image. This vector contains the average grey level, the average contrast, a measure of uniformity and the image entropy. Other descriptors gathered the image texture information using normalised or affine moments. Specifically, the seven moments of $\mathrm{Hu}$ [19] and the six invariant affine moments proposed by Flusser have been obtained [20]. Furthermore, two more feature vectors based on moments have been used. The first one contains nine values coming from the Legendre polynomials corresponding to the five first moments from orders 0 to 2 and the three last moments of the orders 3 and 4 . The fifth feature vector for global texture, a 27-dimensional descriptor made up of the Zernike orthogonal moments [21] up to the fourth order, which makes nine features; including the real, imaginary and absolute values, it sums up to 27 values.

The two last global texture descriptors evaluated were obtained for both data sets, and they used Haralick's features [5] obtained from the GLCM that is computed on the original image and also on the first level decomposition of the wavelet transform with a Haar mother function. Using the five matrices, the original image and the four coefficient matrices from the wavelet decomposition, the two last feature vectors have been computed. Therefore, the sixth global descriptor is a 65-dimensional vector which contains 13 out of the 14 features proposed by Haralick, leaving out just the maximal correlation coefficient. The last global texture description has been carried out, computing just four Haralick features, such as the energy, contrast, correlation and inverse different moment on the original greyscale image and the first wavelet decomposition, yielding a 20-dimensional feature vector.

\section{Local binary pattern}

LBP presented by Ojala et al. [7] is a greyscale texture descriptor that extracts the local spatial structure of an image. Given a pixel, a pattern code is computed by comparing this pixel with the value of its neighbours: 


$$
\mathrm{LBP}_{P, R}=\sum_{p=0}^{P-1} s\left(g_{p}-g_{c}\right) 2^{p}, s(x)=\left\{\begin{array}{ll}
1 & \text { if } x \geq 0 \\
0 & \text { if } x<0
\end{array},\right.
$$

where $g_{c}$ is the value of the central pixel, $g_{p}$ is the value of its neighbour $p, P$ is the number of neighbours and $R$ is the radius of the neighbourhood.

After LBP is obtained for each pixel, in this work, a histogram is built in order to describe the whole image using $P+2$ bins, yielding the feature vector of the image. The pattern extraction process for 1 pixel is shown in Figure 1.

\section{Adaptive local binary pattern}

In their study [11], Guo et al. presented an adaptive descriptor based on local binary pattern motivated by the lack of information about the orientation in the local binary pattern approach. In their work, the oriented mean and standard deviation of the local absolute difference are taken into account in order to make the matching more robust against local spatial structure changes. To minimise the variations of the mean and standard deviation of the directional differences, Guo et al. proposed a scheme that minimises the directional difference $\left|g_{c}-w_{p} * g_{p}\right|$ along different orientations adding the parameter $w$. The objective function is defined as follows:

$$
w_{p}=\arg _{w} \min \left\{\sum_{i=1}^{N} \sum_{j=1}^{M}\left|g_{c}(i, j)-w \cdot g_{p}(i, j)\right|^{2}\right\},
$$

where $w_{p}$ is the weight element used to minimise the directional difference, and $N$ and $M$ are the number of rows and columns in the image, respectively. Each weight $w_{p}$ is estimated along one orientation $2 p \pi / P$ for the whole image.

In their work, Guo et al. used least squares estimation technique to obtain the optimum weight parameter vector $(w)$. After all, the ALBP method is defined as follows:

$$
\operatorname{ALBP}_{P, R}=\sum_{p=0}^{P-1} s\left(g_{p}-w_{p} \cdot g_{c}\right) 2^{p}, s(x)=\left\{\begin{array}{ll}
1 & \text { if } x \geq 0 \\
0 & \text { if } x<0
\end{array} .\right.
$$

\section{Proposed method: ALBPS}

Guo et al. [11] proposed the use of the oriented mean and standard deviation in the matching algorithm to improve classification performance. However, the ALBP method proposed by them does not take into account these statistical values to compute the image descriptor; instead, they were only applied to minimise the directional difference along the different orientations using the weight parameter $w_{p}$. Our proposal includes the standard deviation information not in the matching method but in the descriptor algorithm, and it is called ALBPS on that account. Furthermore, whereas a $1 \times 1$ matching technique was proposed by Guo et al., our scheme uses the support vector machine algorithm in order to classify a descriptor. This is a huge advantage in most cases because, in this way, it is possible to use a fast and powerful classifier that will perform very well when the training set is big enough.

The standard deviation vector $\sigma$ is obtained using Equation 4:

$$
\sigma_{p}=\sqrt{\sum_{i=1}^{N} \sum_{j=1}^{M}\left(g_{c}(i, j)-g_{p}(i, j)-\mu_{p}\right)^{2} /(M \cdot N)},
$$

where $N$ and $M$ are the numbers of rows and columns respectively, $g_{c}(i, j)$ is the centre pixel at position $(i, j)$, $g_{c}(i, j)$ is the neighbourhood of $g_{c}(i, j)$ lying along the orientation $2 p \pi / P$ with the radius $R$ and $\mu_{p}$ is the oriented mean obtained using the following:

$$
\mu_{p}=\sum_{i=1}^{N} \sum_{j=1}^{M}\left|g_{c}(i, j)-g_{p}(i, j)\right| /(M \cdot N)
$$

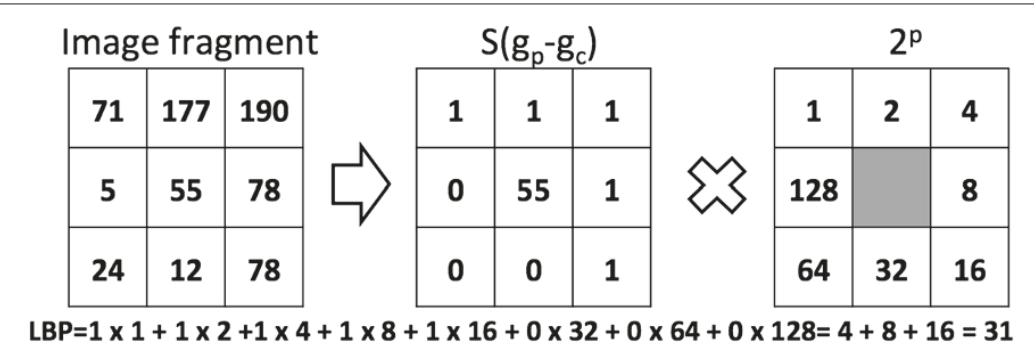

Figure 1 Local binary pattern process. Local binary pattern (LBP) process over one greyscale pixel with $P=8$ and $R=1$. LBP code assigned to the central pixel is calculated by multiplying the output of the threshold function by the term $2^{p}$ for each neighbour pixel and then summing all those values. 

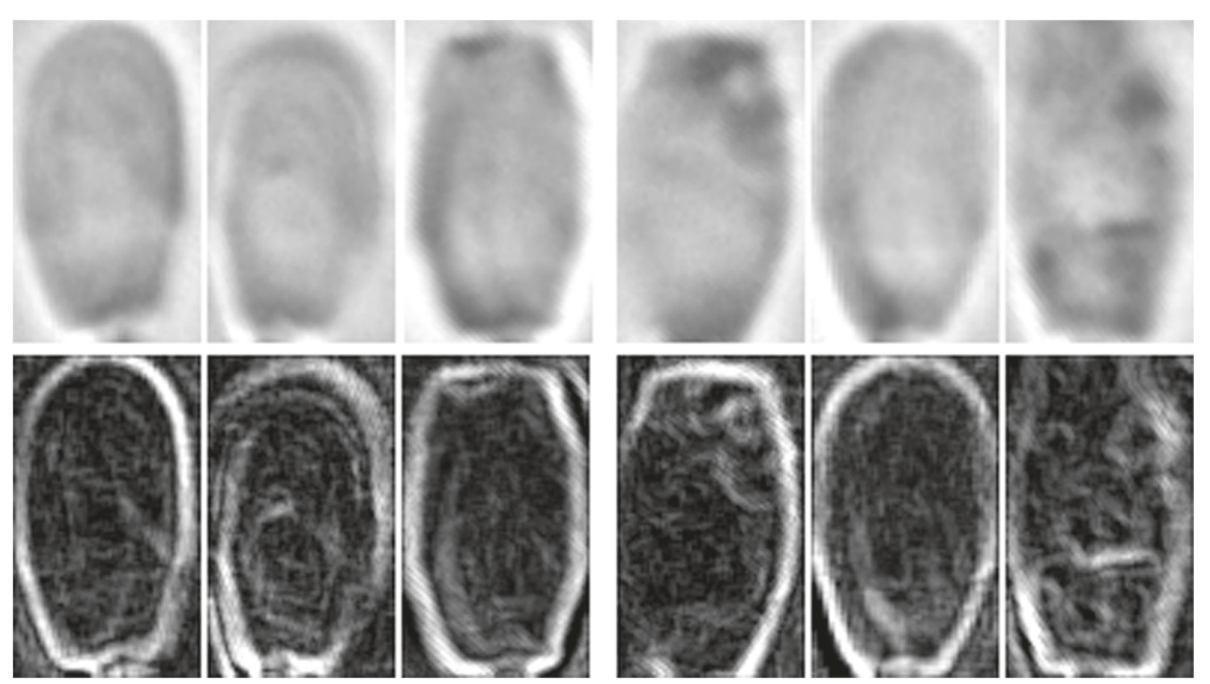

Figure 2 Examples of spermatozoa head images. The first row shows registered greyscale heads and the second row their range filtered outputs. The first three images are examples of alive heads and the last three of dead ones.

ALBPS descriptor is obtained by concatenating the $P+$ 2 bin histogram values of the uniform LBP approach together with the $P$-dimensional standard deviation vector, yielding a descriptor of $2 P+2$ features with $P$ as the size of the neighbourhood.

The standard deviation was chosen as oriented statistical feature because it can reflect the high difference of homogeneity seen in the dead heads (heterogeneous texture) in contrast with the alive ones (homogeneous texture). We preferred this statistic rather than the mean because, sometimes, dead heads present black and white dots that can be counteracted when calculating the mean value. Go to the first row of Figure 2 to see examples of dead and alive spermatozoa heads.

\section{Experiments \\ Data sets}

Boar spermatozoa data set

The lack of publicly available data sets of dead and alive boar sperm images forced us to collect our image data set. These images have been captured in CENTROTEC, an artificial insemination centre that is a University of Leon spin-off, and the sperm was obtained from three different races of boars: large white, Piyorker and Landrace. A total of 450 pairs of images have been captured using a Nikon Eclipse microscope (Nikon Co., Shinjuku, Tokyo, Japan) and a Basler A312f camera (Basler Electric Company, Highland, IL, USA) of progressive scan obtaining 351 dead and 450 alive spermatozoa head images. These pairs contain one image in positive phase contrast, and its fluorescent image was obtained using two different stains: propidium iodide dyes dead spermatozoa to red, and dichlorofluorescein dyes the alive spermatozoa green. We encourage the reader to see more about the sample preparation in the study of Sanchez et al. [22]. We have captured the phase contrast images for developing and testing the texture descriptors evaluated on the proposed method. The fluorescent images were used to obtain the ground truth in order to label all the heads in the data set. Examples of these captures can be shown in Figure 3.

After labelling all the images, each head has been automatically registered in order to assure scale and rotation invariance. First of all, the heads have been rotated to its vertical position. This is performed by relating any
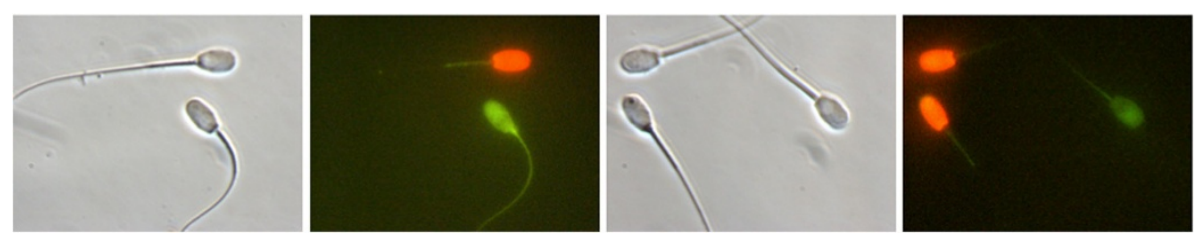

Figure 3 Boar spermatozoa images. Two pairs of images captured. In the left of each pair are the greyscale images and in the right are the fluorescent ones. 

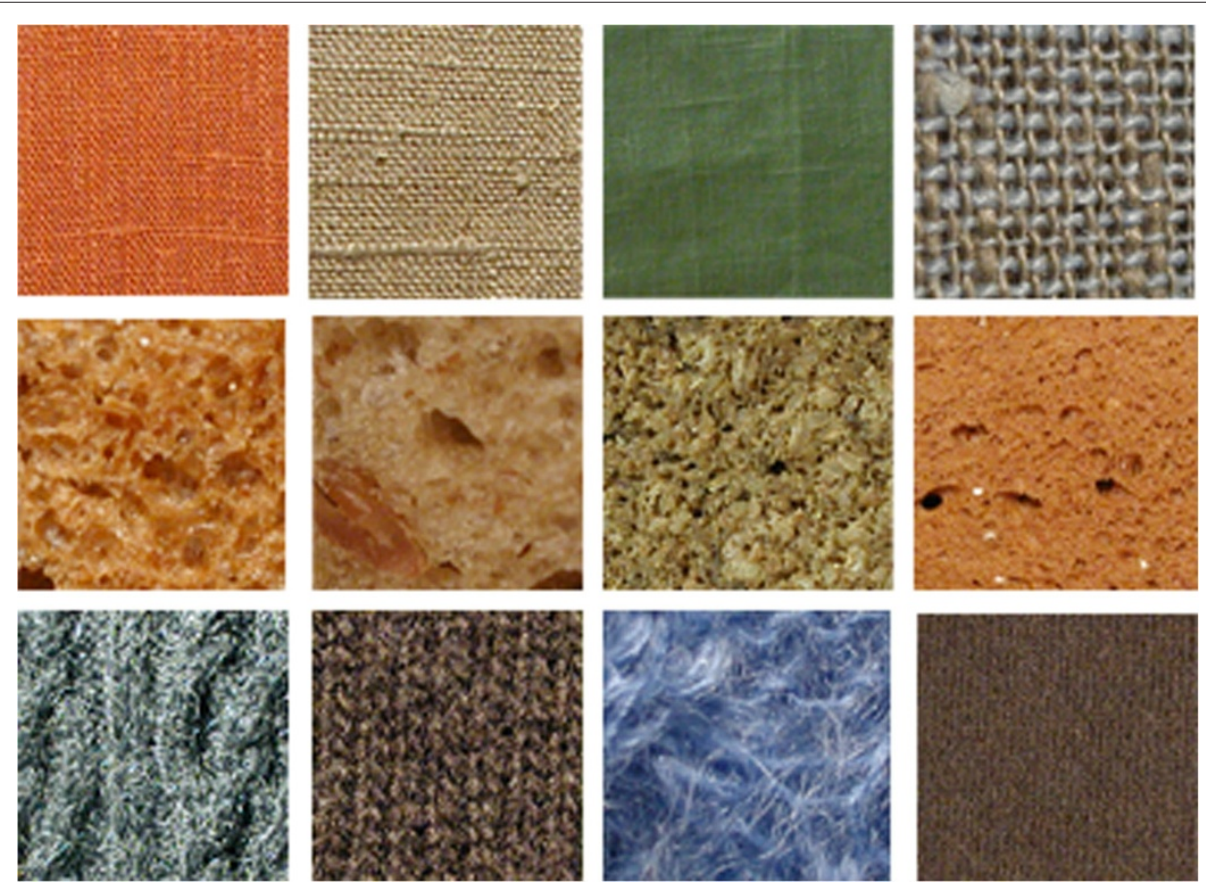

Figure 4 Examples of KTH-TIPS 2a data set. In the first row are different images of the aluminium texture. In the second row are corduroy images, and in the third row are lettuce leaves under different scales and illumination.

sperm head with an ellipse and correcting the orientation of the major axis to achieve verticality, and then the image has been cropped right and left, leaving head pixels untouched. Afterwards, the tail coordinates has been detected. Evaluating if the tail is placed at the bottom half or at the top half of the image will let us know if the spermatozoon has its head up or down, respectively. In the second case, the image has been flipped, leading to equal orientations. The image has been cropped up and down, leaving head pixels intact.

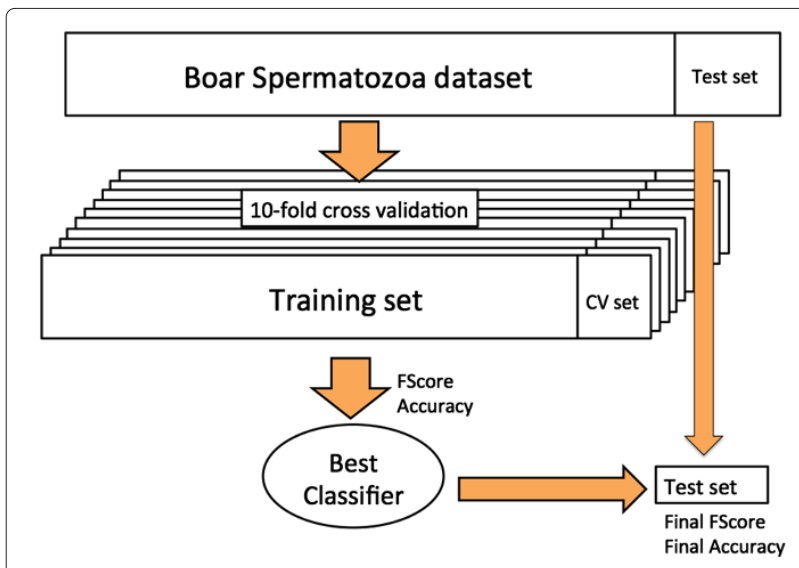

Figure $\mathbf{5}$ Scheme of the experimental set-up. The image shows the experimental set-up carried out using a cross validation set.
Finally, a $3 \times 3$ texture range filter has been applied over the whole data set in order to reduce the noninformative areas and therefore facilitate the subsequent data set description and classification. Figure 2 shows greyscale dead and alive heads and their filtered outputs.

\section{KTH-TIPS 2a data set}

This data set is composed of several images for material categorization [15]. It contains 11 materials (aluminium, brown bread, lettuce, wool, white bread, corduroy, cracker, linen, cork, wood and cotton) with a number of images for four different samples from each material. All the samples were taken at nine scales and three poses under four different illumination conditions. All this variations make a very challenging data set. In Figure 4, we can see some examples of some textures under different conditions.

\section{Experimental set-up Boar spermatozoa data set}

Once the 450 images were range-filtered and the ground truth vector was obtained using the fluorescent images, a cross validation algorithm had been implemented in order to avoid biased results. First of all, a $20 \%$ random subset of the total number of images is kept back in order to get the test results. With the rest of the images, a tenfold cross validation has been carried out. Classification was accomplished using SVM with least squares training algorithm. 
Table 1 Performance of global and local texture descriptors using spermatozoa data set

\begin{tabular}{lccccccccccc}
\hline & \multicolumn{1}{c}{} & \multicolumn{4}{c}{ Global texture descriptors } & \multicolumn{4}{c}{ Local texture descriptors } \\
& WCF13 & WCF4 & Statistical & Legendre & Flusser & Zernike & Hu & ALBP16 & ALBP8 & LBP8 & LBP16 \\
\hline F score & 0.800 & 0.795 & 0.783 & 0.780 & 0.739 & 0.736 & 0.720 & 0.737 & 0.683 & 0.674 & 0.603 \\
Precision(\%) & 80.43 & 79.54 & 78.72 & 71.00 & 75.58 & 71.38 & 66.24 & 73.26 & 67.47 & 67.05 & 60.00 \\
Recall(\%) & 79.57 & 79.54 & 78.10 & 86.59 & 72.65 & 76.29 & 78.76 & 74.12 & 69.14 & 67.82 & 60.67 \\
Accuracy(\%) & 76.88 & 76.75 & 75.69 & 75.00 & 70.88 & 70.19 & 65.44 & 71.88 & 67.50 & 64.38 & 55.63 \\
\hline
\end{tabular}

Performance of global (left) and local (right) texture descriptors LBP and ALBP using $R=1, P=8$ and $R=2, P=16$ asneighbourhoods.

SVM methods are, in general, more efficient in terms of time than the nearest neighbour algorithms. For that reason and the fact that the nearest neighbour algorithm is prone to over-fitting because of its highly non-linear nature, we have selected SVM as our classifier.

Since this data set is skewed due to the high number of alive heads in contrast with the low number of dead ones, $F$ score has been used as one of the quality metrics, and it was applied over the skewed class, in this case, the dead image subset. $F$ score has been computed as $F$ score $=2 \cdot$ precision $\cdot$ recall $/($ precision + recall $)$, where precision $=\mathrm{TP} /(\mathrm{TP}+\mathrm{FP})$ and recall $=\mathrm{TP} /(\mathrm{TP}+\mathrm{FN})$, with TP being the number of true positives in the classification, TN being the number of true negatives, and FP and FN being the false positives and false negatives, respectively. Note that the positive class is the one with less elements in the training set; in this instance, the positive class corresponds to the dead heads. $F$ score results are in the range $[0,1]$ where values near 0 indicate a poor classification and values close to 1 show a good performance.

As the process of selecting the training and cross validation subsets is random, sometimes, the number of images in each class could be quite balanced. For this reason, the accuracy measure has been taken into account and computed as accuracy $=(\mathrm{TP}+\mathrm{TN}) /(\mathrm{TP}+$ $\mathrm{FP}+\mathrm{TN}+\mathrm{FN})$.

Therefore, we obtained $F$ score and accuracy measures for all tenfold combinations of training and cross validation data sets. Afterwards, the classifier which outperformed the others both in terms of accuracy and $F$ score has been selected as the best classifier, and its parameters were used to classify the test set in order to get a more reliable performance. Using a cross validation set instead of a direct test set, we avoid the decision that the best classifier is influenced by the random cross validation set and that the classifier cannot generalise well to future test samples. In Figure 5, a scheme of this process is showed step by step.

\section{KTH-TIPS 2a data set}

The experimental set-up used in this paper for the KTHTIPS 2a data set evaluation is the standard protocol developed by Caputo et al. which was used previously in several papers $[15,16]$. It consists of taking one of the samples of each material for test and the other three for training. This structure yields a more challenging set-up than using random images for test and training. Four classifications using this method have been carried out in order
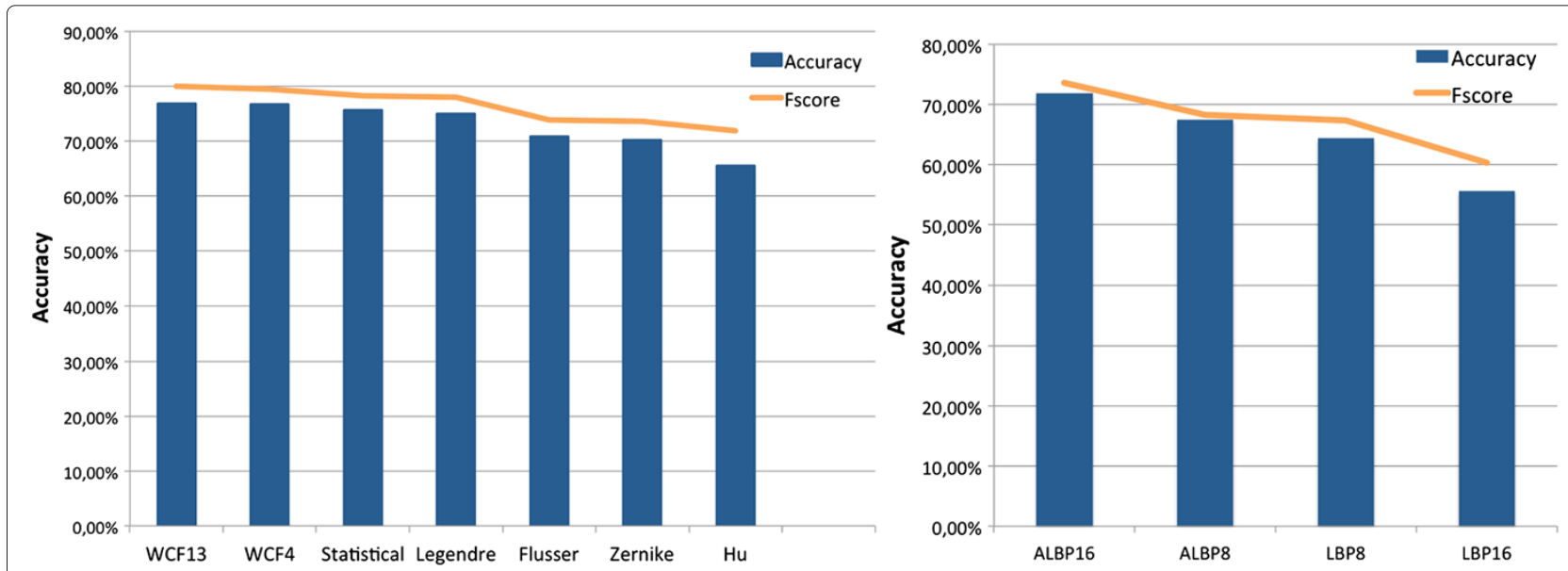

Figure 6 Performance of global and local texture descriptors. Performance of different global texture descriptors (left) and performance of local texture descriptors $L B P$ and ALBP using $R=1, P=8$ and $R=2, P=16$ as neighbourhoods (right). The $F$ score is extended to the range [0-100] in order to preserve the legibility of the graphic. 
Table 2 Performance of our local descriptor using spermatozoa data set

\begin{tabular}{lcccc}
\hline & $\boldsymbol{F}$ score & Precision $(\%)$ & Recall (\%) & Accuracy (\%) \\
\hline ALBPS2,16 & 0.842 & 83.70 & 84.62 & 81.88 \\
ALBPS1,8 & 0.753 & 76.50 & 74.27 & 72.13 \\
LBPS2,16 & 0.747 & 73.61 & 76.17 & 70.50 \\
ALBP2,16 & 0.737 & 73.26 & 74.12 & 71.88 \\
LBPS1,8 & 0.710 & 71.07 & 71.10 & 69.25 \\
\hline
\end{tabular}

Performance of our proposed texture descriptors compared with the best of previous local texture descriptors, ALBP2,16.

to increase its robustness using one sample for test in each iteration. The mean of the hit rate in each classification was computed. We have used support vector machine to classify the images with the one-vs-all paradigm and have selected the least squares training algorithm and a linear kernel to find the decision boundary due to the characteristics of the data set.

\section{Results using sperm data set}

As it was explained in the previous section, in our first approach, several well-known global texture descriptors have been evaluated. In Table 1 (left), it is possible to see the $F$ score, precision, recall and accuracy achieved with these descriptors, whereas in Figure 6 (left), the $F$ score and accuracy results and how they are directly related are shown graphically.

As it can be noticed, using wavelet concurrent feature 13 (WCF13), the performance improved compared to the rest of the global descriptors, yielding both the best $F$ score value and the best accuracy. In contrast, values from $\mathrm{Hu}$, Zernike and Flusser moments are quite low; $\mathrm{Hu}$ just obtained a $65 \%$ of accuracy which is an unacceptable result for this problem. Therefore, we can conclude that global texture descriptors offer poor results for assessing the vitality of boar semen samples.

In the second tests, we used the local texture descriptors LBP and the adaptive version ALBP proposed by Guo et al. [11]. Two different neighbourhoods, $R=1, P=8$ and $R=2, P=16$ have been used to measure $F$ score and accuracy in both cases. Their performance can be seen in Table 1 (right) and Figure 6 (right).

ALBP behaves better than LBP in all cases. ALBP2,16 obtains similar results to global descriptors, with an $F$ score $=0.737$ and an accuracy of $71.88 \%$, outperforming in more than $15 \%$ the accuracy of the classical LBP2,16. Nevertheless, global WCF13 descriptor outperforms the ALBP approach.

In the next experiment, the performance using our proposed method, ALBPS, which includes oriented standard deviation information for the image description has been assessed. To measure the performance of our proposal, we compared it with LBP and ALBP methods. Results when concatenating LBP histogram from the original LBP method with oriented standard deviation vector (LBPS) have also been obtained.

In Table 2 and Figure 7 (left), a comparison between ALBP2,16 ,i.e. the best previous local texture descriptor, and our proposed descriptors is shown. Adding oriented standard deviation to local texture descriptors, LBPS and ALBPS, improves both the original LBP and ALBP methods which verifies the effectiveness of our proposal. The best overall result is achieved with ALBPS with $R=$ $2, P=16$ (ALBPS2,16), yielding an $F$ score $=0.842$ and an accuracy of $81.88 \%$ which means an improvement of $14.25 \%$ in the $F$ score and $13.91 \%$ in accuracy over the base method, ALBP2,16. Moreover, we would like to
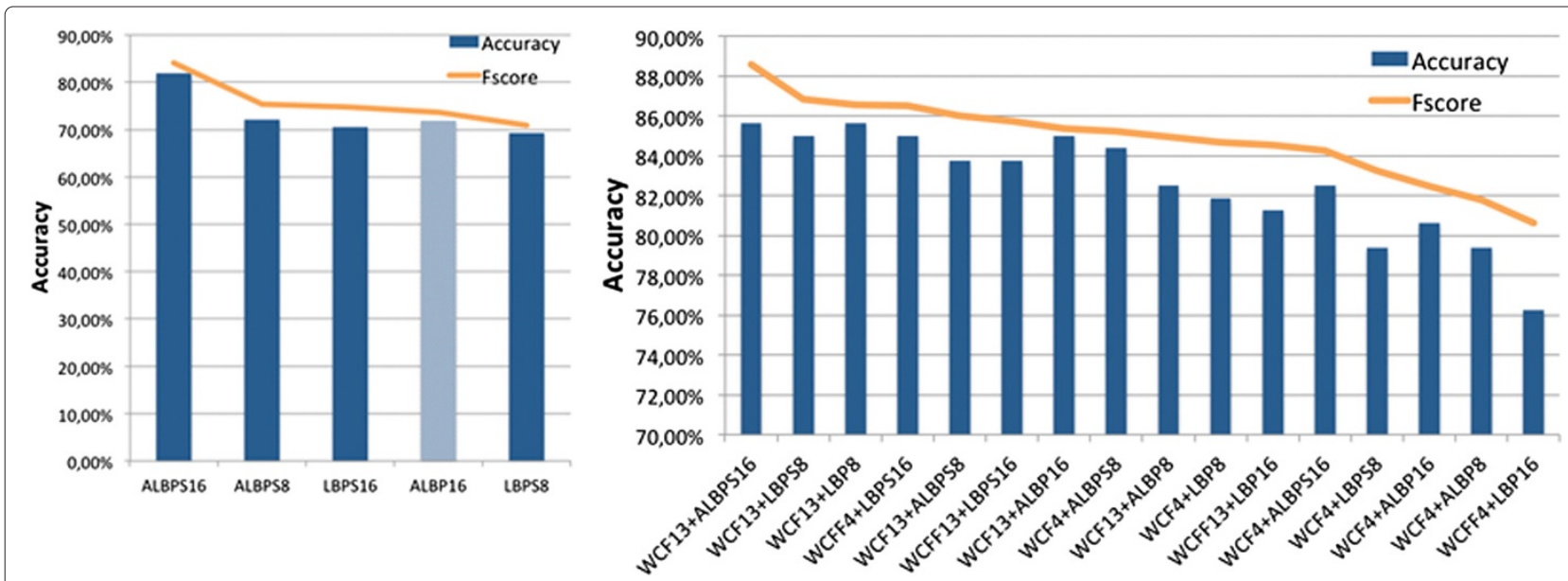

Figure 7 Performance of our proposed local descriptors. Performance of our proposed texture descriptors and the best of previous local texture descriptors ALBP2,16 (dotted bar) (left) and performance of hybrid global and local texture descriptors (right). The $F$ score is extended to the range [0-100] in order to preserve the legibility of the graphic. 
Table 3 Performance of hybrid global and local texture descriptors using spermatozoa data set

\begin{tabular}{|c|c|c|c|c|}
\hline & $F$ score & Precision (\%) & Recall (\%) & Accuracy (\%) \\
\hline WCF13+ALBPS16 & 0.886 & 88.12 & 89.00 & 85.63 \\
\hline WCF13+LBPS8 & 0.867 & 89.77 & 84.04 & 85.00 \\
\hline WCF13+LBP8 & 0.865 & 93.67 & 80.43 & 85.63 \\
\hline WCF4+LBPS16 & 0.865 & 87.50 & 85.56 & 85.00 \\
\hline WCF13+ALBPS8 & 0.860 & 84.21 & 87.91 & 83.75 \\
\hline WCF13+LBPS16 & 0.857 & 80.41 & 91.76 & 83.75 \\
\hline WCF13+ALBP16 & 0.854 & 86.42 & 84.34 & 85.00 \\
\hline WCF4+ALBPS8 & 0.852 & 87.80 & 82.76 & 84.38 \\
\hline WCF13+ALBP8 & 0.850 & 85.87 & 84.04 & 82.50 \\
\hline WCF4+LBP8 & 0.847 & 86.02 & 83.33 & 81.88 \\
\hline WCF13+LBP2,16 & 0.845 & 82.83 & 86.32 & 81.25 \\
\hline WCF4+ALBPS16 & 0.843 & 84.27 & 84.27 & 82.50 \\
\hline WCF4+LBPS8 & 0.832 & 81.19 & 85.42 & 79.38 \\
\hline WCF4+ALBP16 & 0.825 & 82.02 & 82.95 & 80.63 \\
\hline WCF4+ALBP8 & 0.818 & 76.29 & 88.10 & 79.38 \\
\hline WCF4+LBP16 & 0.806 & 79.80 & 81.44 & 76.25 \\
\hline
\end{tabular}

Performance of our proposed texture descriptors compared with the best of previous local texture descriptors ALBP2,16.

highlight that ALBPS2,16 also outperforms global texture description, specifically, by $5.25 \%$ in $F$ score and by $6.5 \%$ in accuracy with regard to WCF13. It is also noticeable that ALBPS2,16 achieves better results than the previous related works found in the literature. Therefore, it is clear from the experiments that our proposed method outperforms global traditional descriptors, previous local texture descriptors based on LBP and previous related works.

Our last experiment consisted of combining the analysed local descriptors with the best outperforming global features into a new hybrid feature vector. We intended to introduce global context to resolve ambiguities that can occur locally when an image has multiple similar regions. In their study [23], Murphy et al. combined local and global texture for object detection, obtaining a better performance than using only one of them. Consequently, WCF13 and WCF4 were merged with the studied local descriptors, yielding the results shown in Table 3 and Figure 7 (right). The best overall result was achieved when combining WCF13 and ALBPS2,16 with an $F$ score of 0.886 and a $85.63 \%$ of accuracy, outperforming the results obtained with local and global textures separately. Particularly, WCF13+ALBPS2,16 improves the $F$ score value in $5.23 \%$ and accuracy in $4.58 \%$ with regard to our individual local descriptor ALBPS2,16.

It is important to note the high value of recall (89\%) obtained, which means that the algorithm detects a high percentage of dead heads. Specifically, it only misclassifies $11 \%$ of them as alive. Since there are many more images of alive heads than dead ones in our data set, predicting that a new test image is alive has a higher probability of chance than otherwise. A value of recall $=0$ would imply that the algorithm is just classifying all images as alive (no skewed class), and therefore, it is obtaining a high accuracy next to $100 \%$ without being useful for the goal of our task. As a consequence, our approach is correctly classifying the database even with the existence of a skewed class.

Besides, we want to highlight that this hybrid WCF13+ALBPS2,16 descriptor classified with SVM obtains better results than the previous works found in the literature. In the introduction of this paper, we found hit rates of $76.80 \%$ in the works of Alegre et al. $[17,18]$. Therefore, the approach presented in this paper obtains an $8.85 \%$ of improvement over previous works.

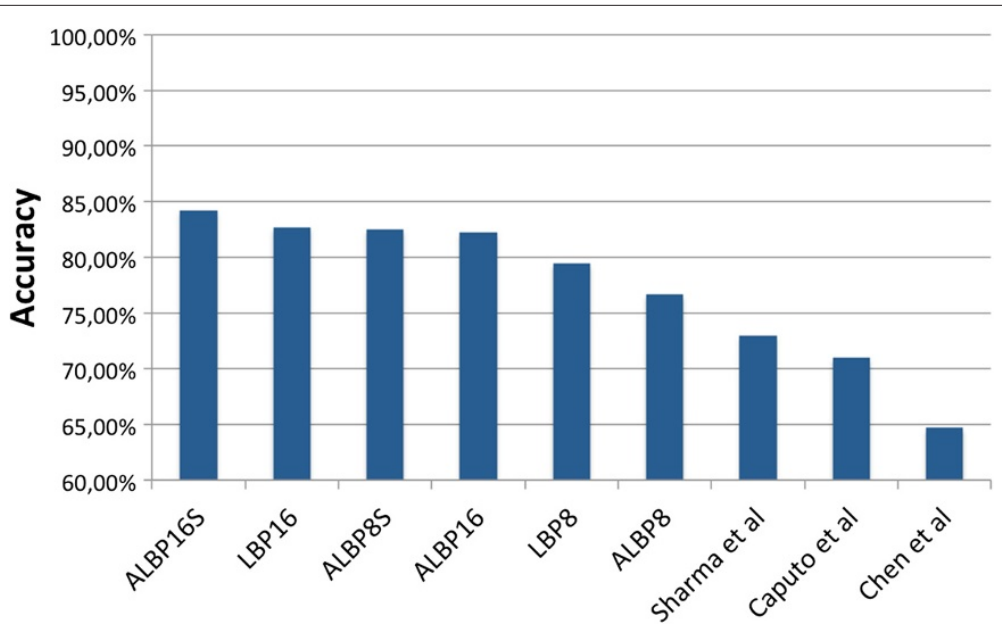

Figure 8 Results achieved on the KTH-TIPS 2a data set. Using KTH-TIPS 2a data set, the best results are obtained using our proposed method ALBPM. 


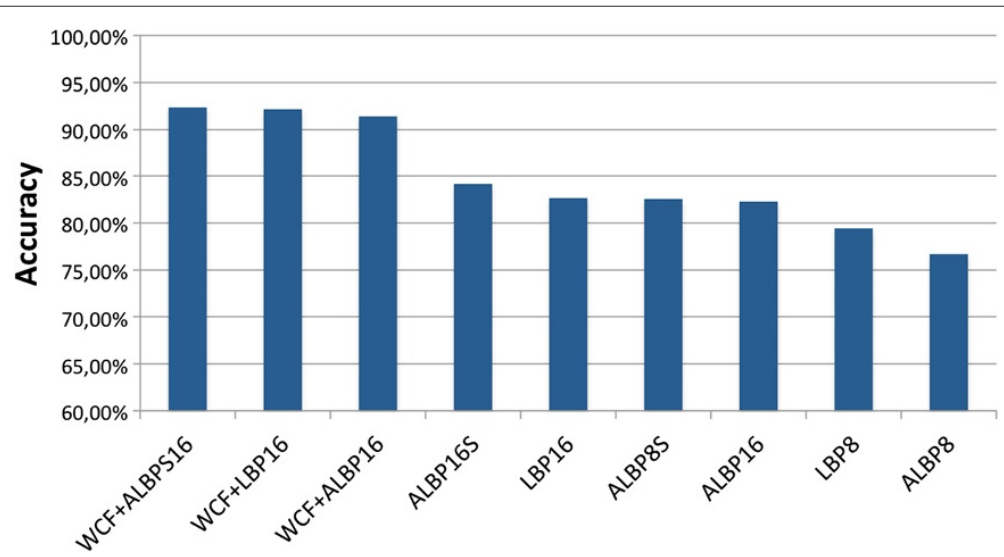

Figure 9 Results using global and local descriptors on the KTH-TIPS 2a data set. The best results are obtained using the proposed method ALBPM combined with WCF, outperforming the local descriptor by themselves.

\section{Results using KTH-TIPS 2a data set}

In this experiment, our proposal has been evaluated using the KTH-TIPS 2a data set, and a comparison with the results achieved in previous works using the same experimental set-up is presented with SVM as classifier. First, Figure 8 shows the results achieved using our improvement of ALBPS and previous results obtained in three recent works by Sharma et al. [24], Caputo et al. [15] and Chen et al. [16]. Our method outperforms all the others, obtaining an improvement of $15.35 \%$ hit rate with respect to the method of Sharma et al., 18.61\% hit rate than the method of Caputo et al. and $30.15 \%$ hit rate than the method of Chen et al.

Secondly, in Figure 9, the local descriptors were combined with the global wavelet-based descriptor reaching an accuracy of $92.36 \%$. Compared to the best local

Table 4 Performance of our proposed method using KTH-TIPS 2a

\begin{tabular}{llc}
\hline Descriptor & & Accuracy \\
\hline Proposed texture descriptors & WCF+ALBPS16 & 92.36 \\
& WCF+LBP16 & 92.14 \\
& WCF+ALBP16 & 91.35 \\
& ALBPS16 & 84.21 \\
& LBP16 & 82.69 \\
& ALBPS8 & 82.53 \\
& ALBP16 & 82.23 \\
& LBP8 & 79.42 \\
Previous works on KTH-TIPS 2a & ALBP8 & 76.64 \\
& Sharma et al. & 73.00 \\
& Caputo et al. & 71.00 \\
& Chen et al. & 64.70 \\
\hline
\end{tabular}

Performance of our proposed texture descriptors compared with previous works on the KTH-TIPS 2a data set. descriptor, ALBPS, the combination of global and local features improves the performance in a $9.68 \%$ hit rate. As we can observe, when merging both global and local descriptors, the results are always better than using only the local descriptors. Our proposed ALBPS method obtained the best results when combined with global descriptors. Table 4 shows the numerical results on KTHTIPS 2a.

\section{Experimental results}

In this subsection, we show the performance evaluation results of the proposed descriptive method in terms of the $F$ score and accuracy using both data sets. All experiments are carried out using support vector machine with least squares and a linear kernel. Experiments using different kernels have been fulfilled, obtaining worse results than with the linear one.

\section{Conclusions}

In this paper, we proposed a new local texture descriptor, ALBPS, by adding an oriented standard deviation term to the ALBP descriptor. It also has been proven that by adding this new term to the classical LBP, its performance also increases. In addition, we have combined the local proposed descriptor, ALBPS2,16, with the global WCF13 descriptor, obtaining a feature vector which contains local and global information. The experimental results showed that the hybrid features extracted by the proposed method provide a better performance than those of previous works when using a robust SVM classification in different data sets. Also, we were able to ascertain that in the spermatozoa data set, the skewed class (the dead one) was successfully classified, reaching a recall of $89 \%$. An $F$ score $=0.886$ and an accuracy of $85.63 \%$ were yielded by WCF13+ALBPS2,16, which is a very interesting result for classifying the vitality of boar spermatozoa 
heads as dead or alive. Furthermore, the results achieved by our method on the KTH-TIPS 2a data set confirm the robustness of our descriptor for general texture classification.

\section{Abbreviations}

ALBP: Adaptive local binary pattern; ALBPS: Adaptive local binary pattern with oriented standard deviation; FN: False negative; FP: False positive; GLCM: Grey level co-occurrence matrix; LBP: Local binary pattern; SVM: Support vector machine; TN: True negative; TP: True positive; WCF: Wavelet concurrent features.

\section{Competing interests}

The authors declare that they have no competing interests.

\section{Authors' information}

OGO was born in León, Spain in 1988. He received a degree in Computer Engineering in 2010 from the University of Leon. In 2011, he received his MSC in Cybernetic Research from the University of León. Currently, he is a PhD student at the Department of Electrical and Systems Engineering at the same university. His research interests include pattern recognition, image processing and face recognition.

EA was born in León, Spain in 1970. He received his Engineering degree from the University of Cantabria, Spain in 1994 and PhD in Computer Sciences from the University of León in 2000. Since 1995, he has been with the University of Leon, Spain, in different positions. Currently, he is an associate professor in Computer Sciences in this university. His research interests focus on image analysis, especially in classification based on texture and shape and in object recognition.

LFR was born in León, Spain in 1985. She received a degree in Industrial Engineering in 2008 from the University of León. In 2011, she received the Certificate in Advanced Studies from the Department of Electrical and Systems Engineering. Currently, she is a PhD student and an assistant teacher on Engineering projects at the same university. Her research interests include object description using local invariant features.

MTGO was born in León, Spain in 1988. She received her Engineering degree from the University of León in 2010, her MSc in Cybernetic Research in 2011 and Masters degree in Training of Secondary, Professional, and Language Teachers in 2012. At present, she is a Ph.D. student and adjunct professor at the Department of Electrical and Systems Engineering, University of León. Her research studies are focused in image processing, texture analysis and shape recognition.

DGO was born in León, Spain in 1985. He received his Computer Engineering degree from the University of Leon in 2010, MSc in Cybernetic Research in 2011 and Masters degree in Training of Secondary, Professional, and Language Teachers in 2012. Currently, he is a PhD student at the Department of Electrical and Systems Engineering. His activity is focused on image robust hashing and video fingerprinting.

\section{Acknowledgements}

This work has been supported by grants DPI2009-08424 and DPI2012-36166 and via the pre-doctoral FPU fellowship program from the Spanish government. The authors would like to thank CENTROTEC for providing us the semen samples and for their collaboration in the acquisition of the images.

\section{Received: 30 November 2012 Accepted: 27 April 2013} Published: 10 May 2013

\section{References}

1. J Zhang, T Tan, Brief review of invariant texture analysis methods. Pattern Recognit. 35(3), 735-747 (2002). [http://www.sciencedirect.com/science/ article/pii/S0031320301000747]

2. Sundaram R, Dhara B, in 3rd International Conference on Electronics Computer Technology (ICECT) 2011, vol. 3, Neural network based Iris recognition system using Haralick features (IEEE Piscataway, 2011), pp. 19-23

3. A Chaddad, C Tanougast, A Dandache, A Bouridane, in 2011 First International Conference on Informatics and Computational Intelligence (ICI), Extraction of Haralick features from segmented texture multispectral bio-images for detection of colon cancer cells (IEEE Piscataway, 2011), pp. 55-59

4. K Keerthana, KThangavel, in 2011 National Conference on Innovations in Emerging Technology (NCOIET), Feature selection in mammogram image using rough set approach (IEEE Piscataway, 2011), pp. 147-152

5. RM Haralick, K Shanmugam, I Dinstein, Textural features for image classification. IEEE Trans. Syst. Man Cybern. 3(6), 610-621 (1973)

6. J Zhang, X Mai, X Wu, in 2011 IEEE International Conference on Computer Science and Automation Engineering (CSAE), vol. 1, Rotation invariant texture classification with dominant orientation estimation based on Gabor filters (IEEE Piscataway, 2011), pp. 606-609

7. TOjala, M Pietikainen, D Harwood, in Proceedings of the 12th IAPR International Conference on Pattern Recognition (ICPR 1994), vol. 1, Performance evaluation of texture measures with classification based on Kullback discrimination of distributions (IEEE Piscataway, 1994), pp. 582-585

8. Y Fang, J Luo, C Lou, in Proceedings of the 3rd international conference on Intelligent information technology application (IITA'09)vol. 2, Fusion of multi-directional rotation invariant uniform LBP features for face recognition (IEEE Piscataway, 2009), pp. 332-335. [http://ieeexplore.ieee. org/stamp/stamp.jsp?tp=\&arnumber=5369391\&isnumber=5367980]

9. S Junding, Z Shisong, W Xiaosheng, in The 2nd IEEE International Conference on Information Management and Engineering, Image retrieval based on an improved CS-LBP descriptor (IEEE Piscataway, 2010), pp. 115-117

10. C Zhu, CE Bichot, L Chen, in International Conference on Pattern Recognition (ICPR), Multi-scale color local binary patterns for visual object classes recognition (IEEE Piscataway, 2010), pp. 3065-3068. [http://liris. cnrs.fr/publis/?id=5033]

11. Z Guo, L Zhang, D Zhang, S Zhang, in 201017 th IEEE International Conference on Image Processing (ICIP), Rotation invariant texture classification using adaptive LBP with directional statistical features (IEEE Piscataway, 2010), pp. $285-288$

12. Z Guo, Q Li, J You, D Zhang, W Liu, Local directional derivative pattern for rotation invariant texture classification. Neural Comput. Appl. 21(8), 1893-1904 (2012)

13. Z Guo, L Zhang, D Zhang, X Mou, in 2010 17th IEEE International Conference on Image Processing (ICIP), Hierarchical multiscale LBP for face and palmprint recognition (IEEE Piscataway, 2010), pp. 4521-4524

14. O Garcia-Olalla, E Alegre, L Fernandez-Robles, MT Garcia-Ordas, in Computer Vision - ACCV 2012 Workshops. ACCV 2012 International Workshops, Daejeon, 5-6 November 2012. Lecture Notes in Computer Science, vol 7728, ed. by JI Park, J Kim. Vitality assessment of boar sperm using an adaptive LBP based on oriented deviation, (Springer Berlin, 2013), pp. 61-72

15. B Caputo, E Hayman, P Mallikarjuna, in Tenth IEEE International Conference on Computer Vision 2005 (ICCV 2005), vol 2, Class-specific material categorisation (IEEE Piscataway, 2005), pp. 1597-1604

16. J Chen, S Shan, C He, G Zhao, M Pietikainen, X Chen, WLD W Gao, in IEEE Transactions on Pattern Analysis and Machine Intelligence, vol 32, A robust local image descriptor (IEEE Piscataway, 2010), pp. 1705-1720

17. E Alegre, O Garcia-Olalla, V Gonzalez-Castro, S Joshi, in Boar spermatozoa classification using longitudinal and transversal profiles (LTP) descriptor in digital images. Proceedings of the 14th International Workshop, IWCIA 2011, Madrid, 23-25 May 2011. Lecture Notes in Computer Science, vol. 6636, ed. by JK Aggarwal, RP Barneva, VE Brimkov, KN Koroutchev, and ER Korutcheva. Combinatorial Image Analysis (Springer Berlin, 2011), pp. 410-419

18. E Alegre, M Garcia-Ordas, V Gonzalez-Castro, S Karthikeyan, in Vitality assessment of boar sperm using NCSR texture descriptor in digital images. Proceedings of the 5th Iberian Conference, IbPRIA 2011, Las Palmas de Gran Canaria, 8-10 June 2011. Lecture Notes in Computer Science, vol. 6669, ed. by J Vitria, JM Sanches, and M Hernandes. Pattern Recognition and Image Analysis (Springer Berlin, 2011), pp. 540-547

19. MK Hu, Visual pattern recognition by moment invariants. IRE Trans. Inf. Theory. 8(2), 179-187 (1962)

20. J Flusser, T Suk, Affine moment invariants: a new tool for character recognition. Pattern Recognit. Lett. 15(4), 433-436 (1994). [http://dx.doi. org/10.1016/0167-8655(94)90092-2]

21. F Zernike, Diffraction theory of the cut procedure and its improved form, the phase contrast method. Physica. 1, 689-704 (1934) 
22. L Sanchez, N Petkov, E Alegre, Statistical approach to boar semen evaluation using intracellular intensity distribution of head images. Cell. Mol. Biol. 52, 38-43 (2006)

23. K Murphy, A Torralba, D Eaton, W Freeman, in Object detection and localization using local and global features in Toward Category-Level Object Recognition. Lecture Notes in Computer Science, vol. 4170, ed. by J Ponce, M Hebert, C Schmid, and A Zisserman (Springer Berlin, 2006), pp. 382-400. [http://dx.doi.org/10.1007/11957959_20]

24. G Sharma, S UI Hussain, F Jurie, in Local higher-order statistics (LHS) for texture categorization and facial analysis, in Computer Vision ECCV 2012. Proceedings of the 12th European Conference on Computer Vision, Florence, 7-13 October 2012. Lecture Notes in Computer Science, vol. 7578 (Springer Berlin, 2012), pp. 1-12. [http://hal.inria.fr/hal-00722819]

doi:10.1186/1687-5281-2013-31

Cite this article as: García-Olalla et al:: Adaptive local binary pattern with oriented standard deviation (ALBPS) for texture classification. EURASIP Journal on Image and Video Processing 2013 2013:31.

\section{Submit your manuscript to a SpringerOpen ${ }^{\circ}$ journal and benefit from:}

- Convenient online submission

Rigorous peer review

- Immediate publication on acceptance

- Open access: articles freely available online

- High visibility within the field

- Retaining the copyright to your article 